\section{Analysis of non-animal methods and models for research in cardiovascular disease}

Emanuele Gasparotti,1,2

Margherita Cioffi, ${ }^{3}$ Vincenzo Positano, ${ }^{1}$

Emanuele Vignali,1,2

Benigno Marco Fanni, 1,2

Katia Capellini, 1,2 Dorela Haxhiademi, ${ }^{4}$

Emiliano Costa, ${ }^{3}$ Sergio Berti, 5

Luigi Landini, 1,2 Simona Celi, 1

Laura Gribaldo 6

1BioCardioLab, Bioengineering Unit,

Fondazione Toscana G. Monasterio,

Massa; 2Department of Information

Engineering, University of Pisa, Pisa;

3RINA Consulting S.p.a, Rome;

${ }^{4}$ Anesthesia and ICU Department, Heart

Hospital, Fondazione Toscana G.

Monasterio, Massa; ${ }^{5}$ Adult Cardiology

Unit, Heart Hospital, Fondazione

Toscana G. Monasterio, Massa, Italy;

6European Commission, DG Joint

Research Centre, Ispra, VA, Italy

\begin{abstract}
Cardiovascular diseases (CVD) are disorders of the heart and blood vessels and represent $31 \%$ of all global deaths. In the contest of CVD, the use of animal experiments has been a contentious subject for many years. In recent years, in vitro and in silico models and methods have been proposed according to the $3 \mathrm{Rs}$ statement. However, an exhaustive report regarding the state of art in terms of in vitro and in silico experiments has not been reported yet. This work is focused on providing a collection of non-animal models and methods in use for basic and applied CVD research. The standardized descriptions of such studies will ultimately feed into EURL ECVAM database on alternative methods. Two are the research main phases. Firstly, the exclusion/inclusion criteria and the list of relevant information resources of the research have been defined. The second phase regards the search, selection and detailed description of the literature papers by analysing records on Scopus and Pubmed databases.
\end{abstract}

\section{Introduction}

Prevalence of cardiovascular disease (CVD) has been increasing worldwide, and the recent report from $\mathrm{AHA}^{1}$ indicates over
90 million US adults have at least one CVD, which is expected to increase more in a rapid pace. In the contest of CVD, the use of animal experiments has been a contentious subject for many years. In recent years, several in vitro and in silico models and methods have been proposed according to the $3 \mathrm{R}$ statement. However, despite a significant amount of literature in the CVD research field, to date, a complete mapping regarding the state of art in terms of efficacy and translational research efficiency is not reported.

In line with such service, the goal of this work is to provide a collection of non-animal models and methods in use for basic and applied CVD research with information on their development status, applications or predictive value in the field of human cardiovascular diseases. The standardized descriptions of such studies will ultimately feed into a EURL ECVAM inventory on innovative methods.

\section{Materials and Methods}

Our research is organized in two main phases: the first phase is dedicated to the setting up of the methodologies, including the exclusion/inclusion criteria and format for the method summaries, the list of relevant information resources and the proposed search phrases. The second phase focuses on the actual performance of the literature search, selection of the methods, analysis and their detailed description. The search was performed analyzing records on Scopus, including Pubmed database.
Correspondence: Simona Celi, BioCardioLab, Bioengineering Unit, Fondazione Toscana G. Monasterio, Massa, Italy.

E-mail: s.celi@ftgm.it

Key words: Cardiovascular diseases; non-animal methods; state of art; in silico; in vitro.

Funding: this project is supported by Tender JRC/IPR/2018/F3/0035/OC grant.

Conference presentation: this paper was presented at the Second Centro 3R Annual Meeting - 3Rs in Italian Universities, 2019, June 20-21, University of Genoa, Italy.

Received for publication: 28 October 2019. Accepted for publication: 11 November 2019.

This work is licensed under a Creative Commons Attribution NonCommercial 4.0 License (CC BY-NC 4.0).

CCopyright: the Author(s), 2019

Licensee PAGEPress, Italy

Biomedical Science and Engineering 2019; 3(s3):112 doi:10.4081/bse.2019.112

\section{Results}

Our preliminary results depict an amount of 14743 research papers on impacted journals in CVD field without the usage of animal models. Cardiovascular Surgical and interventional Procedures (with/without devices) include about 38\% of the records (Figure 1). Regarding the CVD pathologies, the myocardial ischemia is the disease where most of non-animal methods and models are applied.

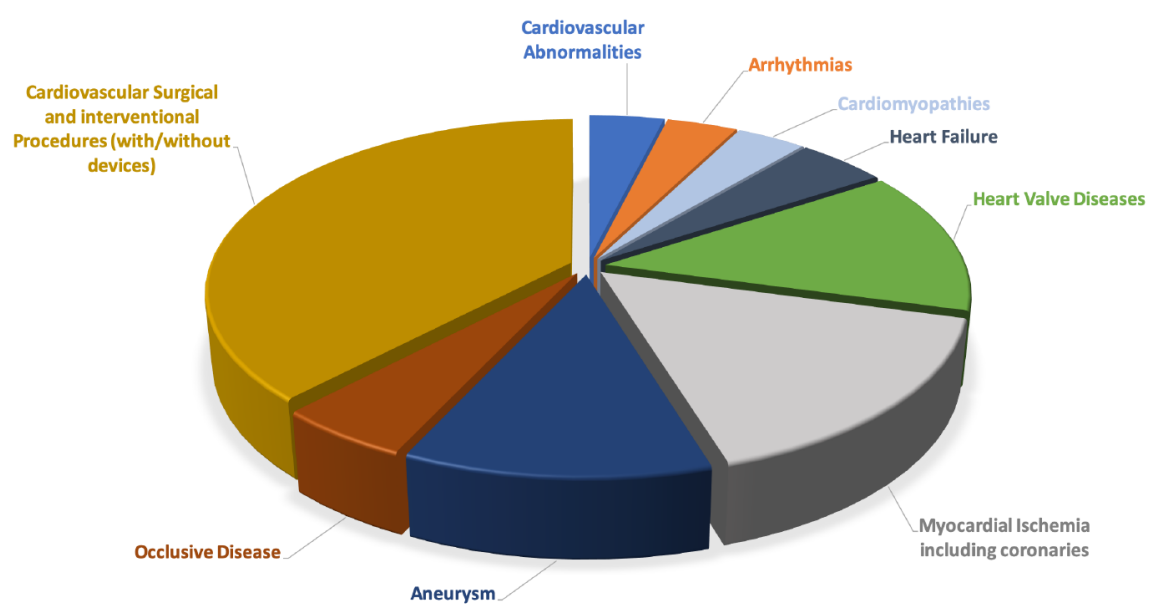

Figure 1. Records categorization. 


\section{Conclusions}

These results seem to be in accordance with the effort of the EU community concerning the past projects in cardiovascular devices and point out fundamental details on further effort by the Community to cope missing research topic. The outcome of this study will be crucial to contribute to the uptake, implementation and promotion of non-animal methodologies in biomedical research, thus contributing to the reduction of the reliance on animal use.

\section{References}

1. Benjamin EJ, Blaha MJ, Chiuve SE, et al. Heart disease and stroke statistics 2017 update: a report from the American Heart Association. Circulation 2017;135:e146-603. 